\title{
Research on operational model of PUBG
}

\author{
Yong Ding \\ Hubei Communications Technical College, Public class department, No. 455 Wuhan Xiong Chu Street, China
}

\begin{abstract}
This paper mainly studies operational model of the famous Computer Games - PlayerUnkown's BattleGrounds (PUBG), be aimed at human beings in the game or human beings regarded as intelligent robots, including the influence of objective factors and subjective factors on the game results, linear programming model is established for the various factors and the game results, using Excel software, realized in different situations the relationship between various factors and the result of game problem solving and plotting table. To solve the problem 1, the paper find out the trend of the safety area and the random coverage circle of the safety area through statistical data and analyze the data, and then push it to the parachute jumping time and the parachute jumping place. To solve the problem 2, the paper build a game model based on the analysis of the distance between two sides, the judgement of the azimuth and the random running and attacking each other caused by the psychological state of the bullet avoidance, and then, according to experience, how to perform micro manipulation to improve the hit rate. To solve the problem 3 , the paper make KDA as a measure of bureau of the game in the best performing team and the best individual (MVP) of the key indicators, through the calculation of KDA for the game and the individual scores and rankings, distribution to determine the winners of the prize gaming contest.
\end{abstract}

\section{Introduction}

PUBG is currently a very popular game, the main content of the game is: 100 game player in the game to take airborne machine to complete the parachute in the island during the flight, each game is divided into several time periods, with the passage of time, the plane on the ground the random bombardment and ground drug on the island will need to continue to spread, game player safety zone in the current travel map and the next time the safety zone and avoid or use the search on the way to the offensive weapons and defense against enemy tools and game player will be able to survive, according to the public security zone will appear during the game time in the random narrow coverage, because the drug was completely covered the island until the fall, players must be in the island before destruction through combat until the survival To the last 1 or the last 1 groups, as a sign of the final victory. In order to win, we need to study it in depth.

Problem one: Predict the random trend of the coverage area in the whole game process, establish a parachute model for the initial time of game, choose the best parachute time and parachute location for 3 people in the game.

Problem two: A model of the situation of the game between the enemy and the enemy is set up, and the quantification of how to operate the micro manipulation can improve the attack hit rate.

Problem three: If the company held WCA games (WCG), your group is the game production company to participate in the work of the trainee or formal staff team, please give a measure of the game in the best performing team and the best individual (MVP) of the key indicators, calculation model of your original group quantitative and accordingly game and individual scores and rankings, distribution to determine gaming contest winners prize.

The purpose of this paper is to analyze the trend of game contraction and the tactics of war by mathematical modeling.

\section{Problem Hypothesis}

1. The game's official data is accurate.

2. In the game, we ignore the player's physical condition, other factors, such as other factors, only consider the factors in the rules of the game.

3. Ignore the changes in the factors in Annex I and regard it as the same.

4 . $R$ - the radius of the outer ring of the safety ring $r-$ the radius of the inner ring of the safety ring.

\section{Model establishment and solution}

\subsection{Research of problem 1}

The first circle is five minutes after the ring, and then five minutes to finish the ring. Second rings, three minutes and twenty seconds after the ring, two minutes and twenty seconds to finish. Third cycles of two minutes and thirty seconds after the ring, one minute and

\footnotetext{
* Corresponding author: ding_yong_maths@126.com
} 
thirty seconds to finish. After fourth cycles and two points, the contraction ring is finished. Fifth cycles and two minutes after the ring, forty seconds to finish. Sixth cycles one minute thirty seconds, thirty seconds to shrink. Seventh cycles one minute thirty seconds, twenty-five seconds to shrink.

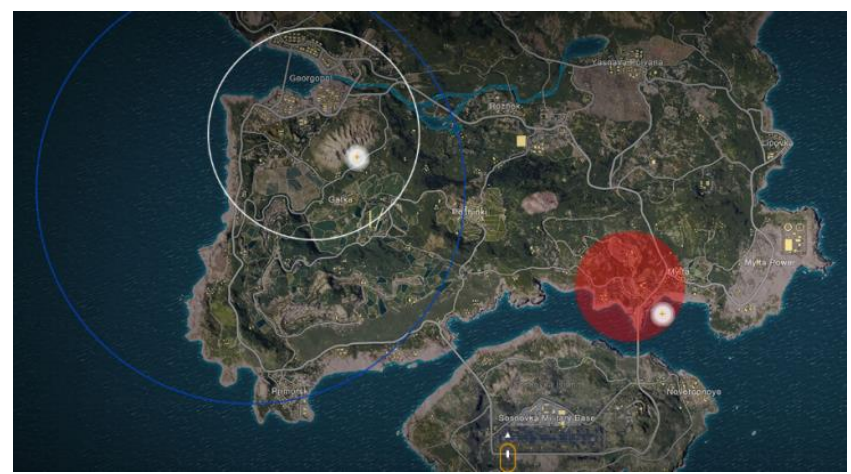

Fig. 1. PUBG Map [1].

The inner ring is slower than the outer ring, and the nearest two points in the blue circle are the slowest, and the farther away the distance is. The size, speed, time and number of the rings have nothing to do with the number.

Table 1. Timing and Damage[2].

\begin{tabular}{|c|c|c|c|c|}
\hline & $\begin{array}{c}\text { Static } \\
\text { timing }\end{array}$ & $\begin{array}{c}\text { Mobile } \\
\text { timing }\end{array}$ & $\begin{array}{c}\text { Damage per } \\
\text { second }\end{array}$ & $\begin{array}{c}\text { Shrinkage } \\
\text { ratio }\end{array}$ \\
\hline 1 & 300 & 300 & 0.4 & 0.4 \\
\hline 2 & 200 & 140 & 0.6 & 0.65 \\
\hline 3 & 150 & 90 & 0.8 & 0.5 \\
\hline 4 & 120 & 60 & 1 & 0.5 \\
\hline 5 & 120 & 40 & 3 & 0.5 \\
\hline 6 & 90 & 30 & 5 & 0.5 \\
\hline 7 & 90 & 30 & 7 & 0.5 \\
\hline 8 & 60 & 30 & 9 & 0.5 \\
\hline
\end{tabular}

From this table, we can see that the second white circle diameter is the first white circle diameter $65 \%$, that is to say, in the first white circle, all possible second white circles all painted, there is a regional intersection, that is to say, when it appeared in the first a white circle, arrived in the intersection area, while second out of the circle will move.

The following figure is the dotted line circle in the center of the large circle, the radius $r$ of the circle is $30 \%$ : $\mathrm{r}=\mathrm{R}(0.65-(1-0.65))=0.3 \mathrm{R}$ of the radius of the large circle.

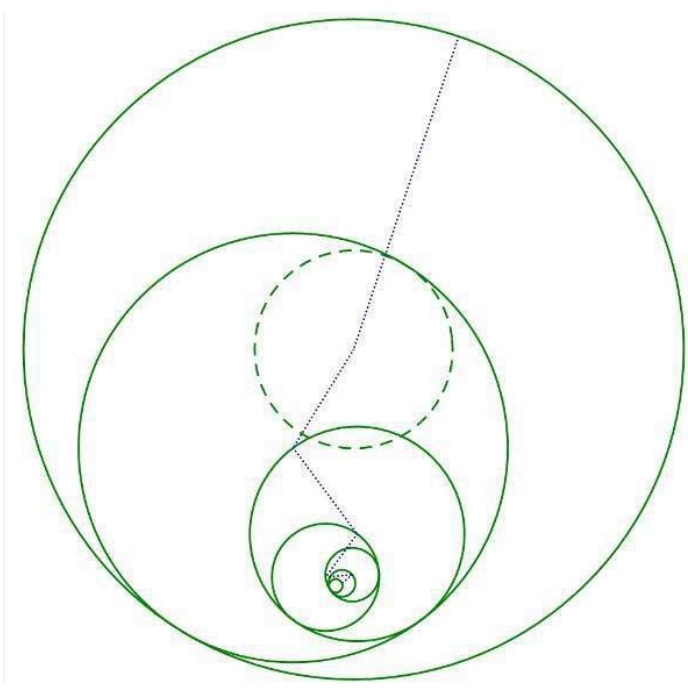

Fig. 2. Further ideas.

Further, we think of each circle as an extreme case, that is, the next circle is the inner circle of the last circle. The shortest path, then, is the center of the first circle to the first circle, the center of the second circle, and the center of the third circle. Use the drawing tools to make all the connections, we can see that the shortest path theory into the circle.

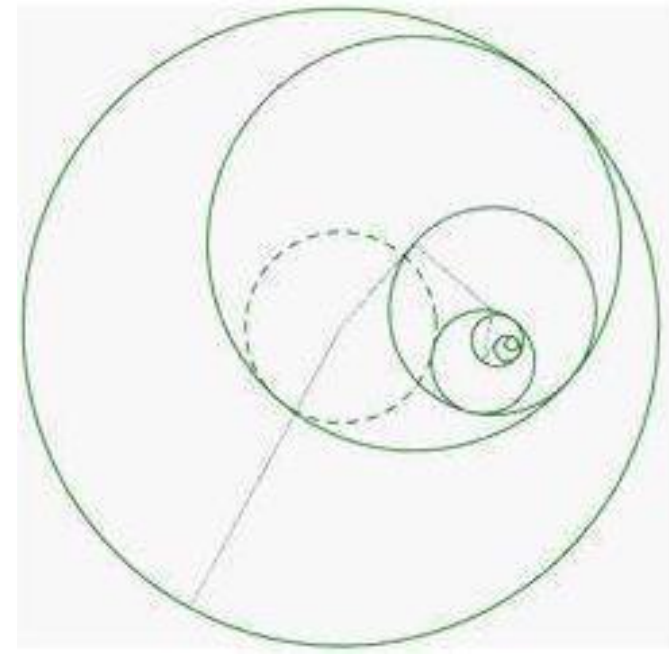

Fig. 3. Extreme case.

As you can see from the figure above, the total line forward distance is a fixed value, the radius $\mathrm{R}$ of the first circle. However, in the actual situation, the choice of the absolute center will be restricted by the terrain or other conditions. Then we can go back and take the "center circle" strategy. That is, when the white circle appears, it is possible to arrive at the region of the next white circle. So when a white circle, even the "scourge circle", also have enough time for tactical planning and tactical maneuver. As Figure 4 shown below, the green dot line circle is the center set of second white circles, and the blue solid line is a possible case of second white circles. The blue dot line circle is the center set of third white circles. The green dashed circle is the intersection area of second white circles and all possible situations. It can also be called the "Golden Circle". The early arrival of the favorable location in the area can ensure that any 
brush ring can make better decisions than those outside the region.

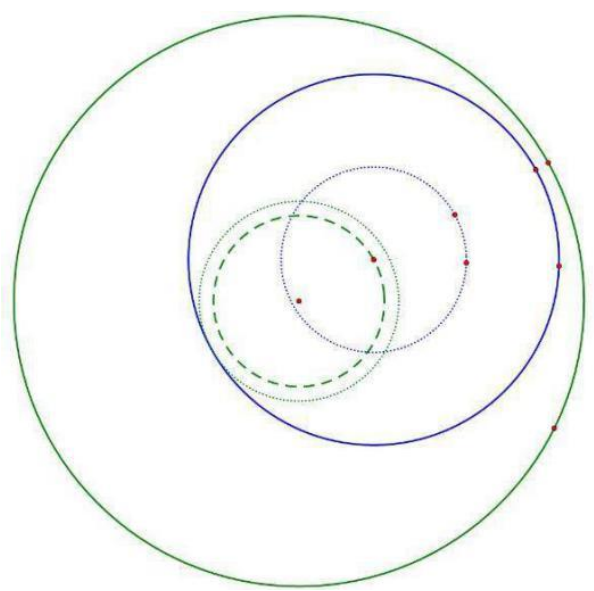

Fig. 4. Gold ring.

In the same level of competition, the center is always better than the clear edge, the time margin of the execution of the tactics is a level.In the game, the full speed state of landing is not horizontal displacement, so it is a full speed state of vertical falling. When full speed, it will glide for a distance, about one hundred meters. In the game, the length of a small white lattice is also one hundred meters. For the best time of parachute jumping and the location of parachute jumping, people should choose the central area parachute. We will adjust it to $200 \mathrm{~m}$ from the target point to about two $(200 \mathrm{~m})$, and then we will adjust it to a goal after diving [3].

\subsection{Research of problem 2}

In the course of the game, we can not avoid fighting against the enemy, which is also one of the key points of victory, and there will be a lot of circumstances in the course of fighting against the enemy.

The process of the war is roughly divided into three categories:

(1) The balance of potential: both sides are not behind the cover or both sides are in the cover, the distance is not far away, can quickly find each other in the direction of the attack.

(2) Advantage duel: the first step to find the other, the first after the cover, the first attack.

(3) Inferior duel: after being removed from the bunker, it is discovered by the enemy first, or is unable to judge the enemy's position after being attacked by the enemy.

If we don't have confidence in our own technology in the balance of power, we should try not to expose the enemy on the outside. At that time, we need to use the surrounding bunkers, terrain and throwing objects to defeat the enemy by tactics. The advantage of the duel, if the enemy and yourself is not far from there can be the first shot, once the enemy stopped towards his teammates or enemies towards yourself immediately retracted, in case of a great chance to strike down or kill each other. In inferior competition, we need to think about whether to run or to fight hard. If we want to run, do not hesitate, but it is hard to run away when there is no bunker around. If we want to fight, we need to be stronger than the other side. In the game to improve the hit rate of micro essential. The face of the opponent, not panic, normally, with two guns, 3-5 guns to parts of the body, then the gun accuracy is very important, don't be afraid to hit the muzzle, head shot slightly under pressure, continuous open two guns, headshot rate, calm is the most important. When you use a sniper gun, you need to lift the gun and calculate the drop of a bullet.

\subsection{Research of problem 3}

In the game DOTA, LOL and hero of Newerth, KDA refers to KILL DEATH ASSIST (killing rate, mortality rate, support rate). Usually KD RATIO (KDR) is used to compare murder rate and mortality rate [4].

In this game PUBG we set the formula as: the number of killings / the number of ground $* 5$, and the default of 1 if the number of ground falls is 0 .

\section{Model evaluation}

Advantage: We have grasped the main factors to solve the problem and abandoned some minor factors, so the answer is simple.

Shortcoming: (1) The problem of the model does not make a specific analysis of sex, thus neglecting the influence of sex, and the completeness of the model is poor. (2) The model can not effectively and intuitively see the relationship between the various factors and the quality of sleep. (3) There may be errors in the data referred to, which leads to the inevitable error in the operation of the data, and the accuracy of the data will be reduced.

Improvement of model: There are various kinds of uncontrollable factors in the game. We can not predict the enemy's actions. Therefore, we need to collect more data in the game to sort out all the factors that may be related to the game results, so that we can better reflect the correctness of the model results.

\section{Next step of research}

The next step of research : Suppose the people is a intelligent robot, the main contents of this paper are multi-objective optimization, including avoiding the best evade routes and methods of poison gas and random bombing, the routes and methods to get the fastest offensive weapons and defensive equipment, and the comprehensive solutions to the above problems. Continuing to improve the mathematical model, introducing matrix is a path.

The firearms, helmet and medicine package of the fastest access route may depend on the beginning of the parachute landing, so I'm going to be on the map in a Cartesian coordinate system, then the points on the map as coordinate points, each point has coordinates, and we will fall in with his teammates placement closer (i.e. the parachute and most of his teammates the same time, to facilitate the team with combat) and a linear distance gun places coordinates (simplified model, the distance 
between two points in the local formula) (i.e. the weighted shortest distance, the two can assume the weight of each accounted for $50 \%$ ).

The coordinate point is placed in a matrix as an element. The direction of the coordinate point leads to the change of the coordinate value of the coordinate point. The change of the coordinate value corresponds to the change of the elements in the matrix, so that MATLAB can be used to process the matrix data. Application examples of this can simulate a genetic algorithm: the shortest path problem of military reconnaissance aircraft reconnaissance base (coordinates of the problem is the coordinates of longitude and latitude, here we will simply map as plane, is Cartesian coordinates rather than spherical coordinate, then our coordinate is horizontal and vertical coordinates).

Further research includes the usage of gold coins [5].

\section{Conclusion}

To solve the problem 1, For the best time of parachute jumping and the location of parachute jumping, people should choose the central area parachute. We will adjust it to $200 \mathrm{~m}$ from the target point to about two $(200 \mathrm{~m})$, and then we will adjust it to a goal after diving.

To solve the problem 2, We have to consider separately from three aspects: The balance of potential, Advantage due, and Inferior duel.

To solve the problem 3 , we set the formula as: the number of killings / the number of ground $* 5$, and the default of 1 if the number of ground falls is 0 .

\section{Acknowledgement}

The research was carried out by the science and technology project of Hubei Provincial Transport Department "the and exploration and practice of Chinese traditional Taoist philosophy cultural integration into modern transportation higher vocational education" (2017-2018), the project of Hubei Communications Technical College "Research on the reform of higher mathematics curriculum in Higher Vocational Education" (2011-2018), and the project of Hubei Communications Technical College "Research on the construction of mathematics test bank for Traffic Vocational Colleges" (2012-2018).

\section{References}

1. Tecent Games. PUBG.

2. http://pubg.qq.com/\#section6 (2018)

3. Yxdown. A detailed analysis of the mechanism of the venom circle in the "PUBG survival". http://www.yxdown.com/gonglue/372025.html (2018)

4. Zhihu. Parachute for the life of the PUBG.

5. https://www.zhihu.com/question/64844816/answer/ 225464286 (2018)
6. Baidu zhidao. What is the meaning of the killing score and the value of life at the end of the extinction interface of the PUBG.

7. https://zhidao.baidu.com/question/52470373414406 8245.html (2018)

8. Crsky. The use of gold coins in the battlefield of PUBG. http://www.crsky.com/zixun/15959.html (2018) 